\title{
Existe associação entre artrite psoriásica e obesidade em adultos?: uma revisão integrativa da literatura
}

A artrite psoriásica (AP) é uma doença musculoesquelética crônica com característica inflamatória imunomediada que se apresenta de forma heterogênea. Ela está relacionada a fatores genéticos, ambientais e estilo de vida, como a obesidade. Nos últimos anos, as taxas de sobrepeso e obesidade aumentaram consideravelmente a ponto de um terço da população ser classificada como com sobrepeso ou obesidade. O objetivo é verificar nas evidências mais recentes a ocorrência entre a AP e a obesidade em adultos. Trata-se de uma revisão integrativa da literatura, na qual foi realizada uma busca de artigos publicados entre os anos 2015-2020 nas bases de dados PubMed e LILACS através dos descritores artrite psoriásica e obesidade nos idiomas inglês, espanhol e português. Dentre os 334 artigos encontrados inicialmente, foram selecionados 12 artigos para compor a amostra final após a aplicação dos critérios de inclusão e exclusão. A obesidade se mostrou frequente e contribuiu para a piora da atividade da doença em pacientes com AP. Além disso, a alimentação baseada em baixa ingesta calórica e cirurgia bariátrica por bypass gástrico mostraram resultados benéficos nesses pacientes. A apresentação tardia da AP também se relacionou a uma maior prevalência da obesidade. As evidências recentes sugerem uma associação comum entre a artrite psoriásica e a obesidade em adultos.

Palavras-chave: Artrite psoriásica; Obesidade; Psoríase.

\section{Is there an association between psoriatic arthritis and obesity in adults?: an integrative literature review}

\begin{abstract}
Psoriatic arthritis (PsA) is a chronic musculoskeletal disease with an immune-mediated inflammatory characteristic that presents in a heterogeneous manner. It is related to genetic, environmental and lifestyle factors such as obesity. In recent years, rates of overweight and obesity have increased considerably to the point that one third of the population is classified as overweight or obese. The objective is checking the most recent evidence the occurrence between PsA and obesity in adults. This article is an integrative literature review. A search for articles published between the years 2015-2020 in the PubMed and LILACS databases was carried out using the keywords: psoriatic arthritis and obesity in English, Spanish and Portuguese. Among the 334 articles found initially, 12 articles were selected to compose the final sample after applying the inclusion and exclusion criteria. Obesity was frequent and contributed to the worsening of disease activity in patients with PsA. In addition, a diet based on low caloric intake and bariatric surgery by gastric bypass have shown beneficial results in these patients. Late presentation of PsA was also related to a higher prevalence of obesity. Recent evidence suggests a common association between psoriatic arthritis and obesity in adults.
\end{abstract}

Keywords: Psoriatic arthritis; Obesity; Psoriasis.

Topic: Reumatologia

Reviewed anonymously in the process of blind peer
Received: 15/02/2021

Approved: 22/03/2021

Tácio Vinícius Almeida Montalvão Barreto (D)

Universidade Tiradentes, Brasil

http://lattes.cnpq.br/1843731968648709

http://orcid.org/0000-0002-6363-636X

taciomedunitix@gmail.com

Denison Santos Silva (iD

Universidade Tiradentes, Brasil

http://lattes.cnpq.br/4938052514549676

http://orcid.org/0000-0002-8518-1757

denisansil@hotmail.com

Referencing this:

BARRETO, T. V. A. M.; SILVA, D. S.. Existe associação entre artrite psoriásica e obesidade em adultos?: uma revisão integrativa da literatura. Scire Salutis, v.11, n.2, p.140-146, 2021. DOI: http://doi.org/10.6008/CBPC2236-9600.2021.002.0016 


\section{INTRODUÇÃO}

A artrite psoriásica (AP) é uma doença musculoesquelética crônica com característica inflamatória imunomediada que se apresenta de forma heterogênea através de sintomas que podem incluir psoríase na pele e unhas, artrite periférica, dactilite, entesite e espondilite . A psoríase é uma manifestação cutânea que afeta $2 \%$ da população mundial e é comumente associada à AP (EDER et al., 2016). A estimativa é que $20 \%$ a $30 \%$ desses pacientes com psoríase irão desenvolver entesite ou artrite ao longo dos anos (TALOTTA et al., 2019). A psoríase geralmente surge antes em aproximadamente $75 \%$ a $84 \%$ dos pacientes por um período que pode variar entre 7 a 12 anos até o desenvolvimento da AP (KAVANAUGH et al., 2018).

O diagnóstico de AP é feito com base nos critérios da Classification of Psoriatic Arthritis (CASPAR). Os pacientes precisam ter doença inflamatória articular (articulação, coluna ou êntese). E pelo menos três pontos entre os seguintes itens: presença de psoríase atual ou histórico sendo o atual como equivalente a dois pontos, distrofia psoriásica em unha, dactilite, ausência de fator reumatoide e evidência radiográfica de nova formação óssea justa-articular (KARREMAN et al., 2016).

A doença está relacionada a fatores genéticos, ambientais e estilo de vida, como a obesidade. Em pacientes obesos, a produção de interleucina-6, fator de necrose tumoral alfa e leptina, por parte dos adipócitos, intensifica o estado inflamatório do organismo, dificultando, assim o controle da AP (BARNAS et al., 2015).

A Organização Mundial da Saúde (OMS) define o sobrepeso e a obesidade como o acúmulo anormal ou excessivo de gordura que apresenta risco para saúde. $\mathrm{O}$ índice de massa corporal (IMC) é uma métrica simples feita através do peso corporal em quilogramas e dividido pela altura elevada ao quadrado em metros. Ele é utilizado para definir sobrepeso ou obesidade quando o IMC é $\geq 25 \mathrm{~kg} / \mathrm{m}^{2}$ e IMC $\geq 30$ $\mathrm{kg} / \mathrm{m}^{2}$, respectivamente (CHOOI et al., 2019).

Nos últimos 35 anos, as taxas de sobrepeso e obesidade aumentaram consideravelmente. Hoje, mais de um terço da população mundial é classificada como com sobrepeso ou obesidade, sendo mais prevalentes em mulheres e com o avançar da idade ( $\mathrm{CHOOI}$ et al., 2019). Diante disso, a pretensão deste trabalho é verificar se há associação entre a AP e obesidade em adultos.

\section{METODOLOGIA}

Trata-se de uma revisão integrativa da literatura composta pelas seguintes etapas: elaboração da pergunta norteadora (existe associação entre a artrite psoriásica e obesidade em adultos?), busca na literatura, filtragem dos dados, apresentação dos resultados e interpretação das informações coletadas.

O levantamento dos artigos foi realizado na base de dados PubMed e LILACS. Os descritores utilizados foram "Artrite psoriásica" e "Obesidade" consultados na plataforma DeCS nos idiomas inglês, espanhol e português. Para o cruzamento dos descritores foram utilizados os termos boleanos "AND" e "OR". Foi selecionado o campo de busca "all fields" no PubMed e "Título, resumo, assunto" no LILACS. A aplicação dos descritores foi feita da seguinte forma: (Artrite Psoriásica AND Obesidade) OR (Artritis Psoriásica AND Obesidad) OR (Arthritis, Psoriatic AND Obesity). 
Os critérios de inclusão para o estudo foram os artigos publicados em idioma inglês, espanhol ou português nos últimos cinco anos (2015-2020) e de pesquisas em adultos. Foram desconsiderados artigos de relato de caso, periódicos, revisões de literatura, meta-análises e artigos que não se relacionavam à pergunta norteadora.

\section{RESULTADOS E DISCUSSÃO}

No banco de dados PubMed foram encontrados inicialmente 320 artigos através dos descritores supracitados. Após a aplicação dos critérios de inclusão restaram 63 artigos. Em seguida, foram aplicados os critérios de exclusão e restaram 12 artigos.

No banco de dados LILACS, ao buscar os descritores, foram encontrados 14 artigos. Após a aplicação dos critérios de inclusão sobrou um artigo e após a aplicação dos critérios de exclusão não restou artigo. A amostra final foi composta por 12 artigos em língua inglesa, dentre eles quatro estudos transversais, sete coortes e um ensaio clínico, reunidos na tabela 1.

Tabela 1. Amostra final dos artigos.

\begin{tabular}{|c|c|c|c|}
\hline Artigo & $\begin{array}{c}\text { Autor/Ano/ Tipo de } \\
\text { estudo }\end{array}$ & Objetivo & Principais resultados \\
\hline $\begin{array}{l}\text { Obesity in psoriatic } \\
\text { arthritis Comparative } \\
\text { prevalence and } \\
\text { associated factors. }\end{array}$ & $\begin{array}{l}\text { (QUEIRO; LORENZO; } \\
\text { TEJÓN; COTO; et al., } \\
\text { 2019)/Transversal. }\end{array}$ & $\begin{array}{l}\text { Analisar a frequência e os fatores } \\
\text { associados a obesidade em uma } \\
\text { coorte de AP. }\end{array}$ & $\begin{array}{l}\text { A obesidade foi mais comum em } \\
\text { pacientes com AP ou psoríase do } \\
\text { que na população sem doença } \\
\text { inflamatória. Sobretudo entre os } \\
\text { pacientes com psoríase sem artrite. }\end{array}$ \\
\hline $\begin{array}{l}\text { The Association } \\
\text { Between Obesity and } \\
\text { Clinical Features of } \\
\text { Psoriatic Arthritis: A } \\
\text { Case-control Study. }\end{array}$ & $\begin{array}{l}\text { (EDER et al., } \\
\text { 2017)/Transversal. }\end{array}$ & $\begin{array}{l}\text { Avaliar se a obesidade está } \\
\text { associada a características distintas } \\
\text { da artrite psoriásica e se ela interage } \\
\text { com os alelos de suscetibilidade ao } \\
\text { HLA da artrite psoriásica }\end{array}$ & $\begin{array}{l}\text { A obesidade foi mais comum entre } \\
\text { aqueles com AP do que os que } \\
\text { tinham psoríase sem artrite. } \\
\text { Maior IMC foi associado com } \\
\text { idades mais avançadas de início da } \\
\text { AP e psoríase. } \\
\text { A obesidade foi mais comum entre } \\
\text { os pacientes com HLA-B27 } \\
\text { negativo. } \\
\text { A obesidade foi menos comum } \\
\text { entre os pacientes com AP que } \\
\text { tinham HLA-B27 positivo. }\end{array}$ \\
\hline $\begin{array}{l}\text { Weight loss improves } \\
\text { disease activity in } \\
\text { patients with psoriatic } \\
\text { arthritis and obesity: on } \\
\text { interventional study. }\end{array}$ & $\begin{array}{l}\text { (KLINGBERG et al., } \\
\text { 2019)/Ensaio clínico. }\end{array}$ & $\begin{array}{l}\text { Estudar prospectivamente os efeitos } \\
\text { do tratamento para perda de peso } \\
\text { com dieta hipocalórica ( } 640 \mathrm{kcal} \text { por } \\
\text { dia) na atividade da doença em } \\
\text { pacientes com AP (critérios CASPAR) } \\
\text { e obesidade (índice de massa } \\
\text { corporal IMC } \geq 33 \mathrm{~kg} / \mathrm{m}^{2} \text { ). }\end{array}$ & $\begin{array}{l}\text { A dieta hipocalórica em pacientes } \\
\text { com AP e obesidade foi associada a } \\
\text { uma melhora significativa no índice } \\
\text { de atividade da doença em } \\
\text { articulações, ênteses e pele. }\end{array}$ \\
\hline $\begin{array}{l}\text { Incidence and } \\
\text { Prognosis of Psoriasis } \\
\text { and Psoriatic Arthritis in } \\
\text { Patients Undergoing } \\
\text { Bariatric Surgery. }\end{array}$ & $\begin{array}{l}\text { (EGEBERG et al., } \\
\text { 2017)/Coorte. }\end{array}$ & $\begin{array}{l}\text { Avaliar a incidência e prognóstico de } \\
\text { pacientes com psoríase e artrite } \\
\text { psoriásica que foram submetidos ao } \\
\text { bypass gástrico ou banda gástrica. }\end{array}$ & $\begin{array}{l}\text { O bypass gástrico foi associado a } \\
\text { uma redução significativa do risco e } \\
\text { melhora prognóstica da psoríase e } \\
\text { AP, enquanto que a banda gástrica } \\
\text { não. }\end{array}$ \\
\hline $\begin{array}{l}\text { Incidence and } \\
\text { Prevalence of } \\
\text { Cardiovascular Risk } \\
\text { Factors Among Patients } \\
\text { With Rheumatoid } \\
\text { Arthritis, Psoriasis, or } \\
\text { Psoriatic Arthritis. }\end{array}$ & $\begin{array}{l}\text { (RADNER et al., } \\
\text { 2017)/Coorte. }\end{array}$ & $\begin{array}{l}\text { Estimar a prevalência e a incidência } \\
\text { de fatores de risco cardiovascular } \\
\text { coma hipertensão, diabetes mellitus, } \\
\text { hiperlipidemia e obesidade em } \\
\text { pacientes com artrite reumatoide } \\
\text { (AR), psoríase ou AP. }\end{array}$ & $\begin{array}{l}\text { As taxas de prevalência e incidência } \\
\text { para hipertensão, diabetes } \\
\text { mellitus, hiperlipidemia e } \\
\text { obesidade foram elevadas entre os } \\
\text { pacientes com AR, psoríase e AP. } \\
\text { Sobretudo, apresentou maiores } \\
\text { índices entre os pacientes com AP. }\end{array}$ \\
\hline
\end{tabular}




\begin{tabular}{|c|c|c|c|}
\hline $\begin{array}{l}\text { Association of central } \\
\text { adiposity with psoriasis, } \\
\text { psoriatic arthritis and } \\
\text { rheumatoid arthritis: a } \\
\text { cross-sectional study of } \\
\text { the UK Biobank. }\end{array}$ & $\begin{array}{l}\text { (FERGUSON et al., } \\
\text { 2019)/Transversal. }\end{array}$ & $\begin{array}{l}\text { Determinar a associação } \\
\text { independente da adiposidade } \\
\text { central, avaliada pela circunferência } \\
\text { da cintura, com a probabilidade de } \\
\text { prevalência para psoríase, AP e AR } \\
\text { após o controle da adiposidade geral } \\
\text { (IMC). }\end{array}$ & $\begin{array}{l}\text { Circunferência de cintura mais } \\
\text { elevada foi associada a maior } \\
\text { probabilidade de psoríase, artrite } \\
\text { psoriásica e artrite reumatoide. }\end{array}$ \\
\hline $\begin{array}{l}\text { Bariatric Surgery and } \\
\text { the Incidence of } \\
\text { Psoriasis and Psoriatic } \\
\text { Arthritis in the Swedish } \\
\text { Obese Subjects Study. }\end{array}$ & $\begin{array}{l}\text { (MAGLIO et al., } \\
\text { 2017)/Coorte. }\end{array}$ & $\begin{array}{l}\text { Avaliar o efeito da cirurgia bariátrica } \\
\text { (gastroplastia vertical, banda } \\
\text { gástrica ou bypass gástrico) em } \\
\text { comparação com os cuidados usuais } \\
\text { na incidência de psoríase e AP no } \\
\text { estudo de suecos obesos. }\end{array}$ & $\begin{array}{l}\text { A cirurgia bariátrica está associada } \\
\text { ao menor risco de desenvolver } \\
\text { psoríase comparado com os } \\
\text { tratamentos usuais. Entretanto, } \\
\text { não houve diferença significativa } \\
\text { observada para o risco de } \\
\text { desenvolver AP. }\end{array}$ \\
\hline $\begin{array}{l}\text { The influence of obesity } \\
\text { on response to tumour } \\
\text { necrosis factor-a } \\
\text { inhibitors in psoriatic } \\
\text { arthritis: results from } \\
\text { the DANBIO and ICEBIO } \\
\text { registries. }\end{array}$ & $\begin{array}{l}\text { (HøJGAARD et al., } \\
2016) / \text { Coorte. }\end{array}$ & $\begin{array}{l}\text { Investigar o impacto da obesidade } \\
\text { na resposta ao primeiro curso de } \\
\text { tratamento com inibidor de TNF-alfa } \\
\text { (I-TNF) em pacientes com AP } \\
\text { acompanhados em cuidados de } \\
\text { rotina. }\end{array}$ & $\begin{array}{l}\text { A obesidade foi associada a uma } \\
\text { maior atividade da doença e } \\
\text { pareceu diminuir a resposta bem } \\
\text { como a adesão ao I-TNF em } \\
\text { pacientes com AP. }\end{array}$ \\
\hline $\begin{array}{l}\text { Higher Incidence Rates } \\
\text { of Comorbidities in } \\
\text { Patients with Psoriatic } \\
\text { Arthritis Compared } \\
\text { with the General } \\
\text { Population Using U.S. } \\
\text { Administrative Claims } \\
\text { Data. }\end{array}$ & $\begin{array}{l}\text { (KAINE et al., } \\
\text { 2019)/Coorte. }\end{array}$ & $\begin{array}{l}\text { Comparar a frequência e as taxas de } \\
\text { incidência de comorbidades e } \\
\text { hospitalizações entre pacientes } \\
\text { recém diagnosticados com AP e uma } \\
\text { população correspondente sem AP } \\
\text { usando grande banco de dados } \\
\text { nacional nos EUA. }\end{array}$ & $\begin{array}{l}\text { Pacientes com AP apresentaram } \\
\text { comorbidades significativamente } \\
\text { elevadas; dentre elas encontramos: } \\
\text { diabetes, ansiedade, fadiga, } \\
\text { tabagismo, uso de álcool, } \\
\text { obesidade ou sobrepeso, } \\
\text { depressão, osteoporose, uveíte, } \\
\text { eczema e gota. }\end{array}$ \\
\hline $\begin{array}{l}\text { Polyarticular evolution } \\
\text { and late-onset psoriasis } \\
\text { may be associated with } \\
\text { cardiovascular disease } \\
\text { in psoriatic arthritis. }\end{array}$ & $\begin{array}{l}\text { (QUEIRO; LORENZO; } \\
\text { TEJÓN; PARDO; et al., } \\
\text { 2019)/Transversal. }\end{array}$ & $\begin{array}{l}\text { Pacientes com AP apresentam alta } \\
\text { prevalência de fatores de risco } \\
\text { cardiovascular (FRC) e também } \\
\text { apresentam taxas mais elevadas de } \\
\text { doenças cardiovasculares (DCV). } \\
\text { Nosso objetivo foi corroborar esses } \\
\text { achados e identificar os fatores } \\
\text { associados a esses eventos no } \\
\text { ambiente clínico. }\end{array}$ & $\begin{array}{l}\text { Pacientes com AP apresentaram } \\
\text { maior frequência de hipertensão, } \\
\text { diabetes, obesidade e tabagismo. }\end{array}$ \\
\hline $\begin{array}{l}\text { Cardiovascular disease } \\
\text { risk profiles in } \\
\text { inflammatory joint } \\
\text { disease entities. }\end{array}$ & $\begin{array}{l}\text { (WIBETOE et al., 2017) } \\
\text { /Coorte. }\end{array}$ & $\begin{array}{l}\text { Comparar os perfis de risco de DCV } \\
\text { em pacientes com doenças } \\
\text { inflamatórias articulares (DIC), } \\
\text { incluindo artrite reumatoide (AR), } \\
\text { espondiloartrite axial (EAA), artrite } \\
\text { psoriásica (AP) e avaliar o risco } \\
\text { futuro de DCV. }\end{array}$ & $\begin{array}{l}\text { Pacientes com AP apresentaram } \\
\text { maiores frequências de } \\
\text { hipertensão e obesidade do que os } \\
\text { pacientes com AR e EAA. }\end{array}$ \\
\hline $\begin{array}{l}\text { Age at disease onset } \\
\text { may help to further } \\
\text { characterize the } \\
\text { diseasephenotype in } \\
\text { psoriatic. }\end{array}$ & $\begin{array}{l}\text { (ALONSO et al., } \\
\text { 2016)/Coorte. }\end{array}$ & $\begin{array}{l}\text { Avaliar se a idade de apresentação } \\
\text { da doença ajuda a caracterizar } \\
\text { melhor o fenótipo da doença na AP. }\end{array}$ & $\begin{array}{l}\text { Houve maior prevalência de } \\
\text { obesidade entre os casos de início } \\
\text { tardio da AP. }\end{array}$ \\
\hline
\end{tabular}

A obesidade foi mais comum entre os pacientes com AP ou psoríase do que entre os pacientes sem apresentação inflamatória. Além do mais, a obesidade foi mais frequente entre os pacientes com psoríase sem artrite (QUEIRO et al., 2019). No entanto, Eder et al. (2017) mostrou resultados diferentes ao observar que a obesidade estava mais associada a AP do que na psoríase sem artrite. Além da obesidade, os pacientes com AP apresentam outras comorbidades com frequência mais elevadas como hipertensão, diabetes e tabagismo (QUEIRO et al., 2019; KAINE et al., 2019).

Ainda, a obesidade foi associada a uma maior atividade da doença na AP do mesmo modo que apresentou menor adesão e resposta ao tratamento de pacientes em uso de I-TNF (HØJGAARD et al., 2016). Ao avaliar a apresentação da AP de forma a considerar início precoce antes dos 40 anos e início 
tardio após essa idade, houve maior prevalência de obesidade entre os casos de início tardio da AP (ALONSO et al., 2016). Outrossim, os maiores valores de IMC foram associados ao início tardio da doença, enquanto que o IMC normal está associado ao início precoce da doença na presença do alelo HLA-B27. Esses achados sugerem um efeito potencialmente diferente da obesidade no risco de AP, dependendo dos fatores genéticos (EDER et al., 2017).

Ferguson et al. (2019) em um estudo do tipo transversal, observou que os pacientes com AP também apresentaram maiores índices de circunferência abdominal de forma pontual, no entanto, ao corrigir a relação entre circunferência abdominal e IMC, não houve ganho estatístico significativo, o que pode indicar que a adiposidade central está associada com maior probabilidade para AR ou psoríase independentemente do IMC, fato que não ocorre na AP. Porém, o grupo de pacientes com AP era consideravelmente menor de modo que pode ter contribuído para reduzir a capacidade de detectar associações significativas em análises ajustadas.

Segundo Klingberg et al. (2019) a dieta hipocalórica pode ser uma nova perspectiva, pois foi notado efeito benéfico na atividade da doença em articulações, ênteses e pele nos pacientes que foram submetidos a uma ingesta calórica com base em $640 \mathrm{Kcal} / \mathrm{dia}$. Esse estudo reforça a hipótese de que a obesidade atua como promotor da atividade da doença na AP.

A cirurgia bariátrica foi avaliada em dois estudos. No primeiro estudo foi feita a comparação entre as técnicas bypass gástrico e banda gástrica. Nele, observou-se que a técnica por bypass gástrico apresentou os melhores efeitos com redução significativa do risco e melhor prognóstico na AP e psoríase. Essa diferença pode ter ocorrido devido à mudança na absorção de nutrientes bem como na secreção de hormônios potencialmente moduladores de inflamação e redução do peso. No bypass gástrico, há um redirecionamento de nutrientes ao contornar um grande segmento do intestino delgado proximal e entrada na parte distal do jejuno diretamente.

Outrossim, há também o aumento na secreção pós-prandial de GLP-1 que é conhecido por reduzir a glicose devido ao seu efeito insulinotrópico e glucagonostático, bem como reduz o apetite ao ativar receptores de GLP-1 no cérebro (EGEBERG et al., 2017). No segundo estudo, a cirurgia bariátrica (gastroplastia vertical, banda gástrica e bypass gástrico) foi comparada aos cuidados habituais e, embora tenha sido notado uma tendência semelhante ao estudo anterior, não foi possível detectar uma redução significativa de AP em indivíduos submetidos à cirurgia bariátrica. Isso pode ter ocorrido devido ao baixo número de participantes com AP. Outra possibilidade é que o papel da obesidade como modulador de risco para a AP não seja tão forte como para a psoríase, visto que neste trabalho a cirurgia bariátrica estava associada ao menor risco de desenvolver psoríase (MAGLIO et al., 2017).

Pacientes com AP também apresentaram maiores frequências de obesidade do que outras condições inflamatórios como AR e espondiloartrite axial (WIBETOE et al., 2017). Em outro estudo que compara os pacientes com AP, AR e psoríase, foi observado que os pacientes com AP também apresentavam maiores frequências de obesidade, o que é particularmente interessante, visto que a maioria dos pesquisadores consideram que os pacientes com AP têm uma carga inflamatória menor do que os 
pacientes com AR (RADNER et al., 2017).

\section{CONCLUSÕES}

As evidências recentes sugerem uma relação comum entre a AP e obesidade. Dessa forma, levanta a possibilidade de novas abordagens que visem o tratamento e acompanhamento em conjunto dessas condições clínicas, de modo a assegurar assistência adequada ao paciente.

\section{REFERÊNCIAS}

ALONSO, S.; TEJÓN, P.; SARASQUETA, C.; COTO, P.; ALPERI, M.; QUEIRO, R.. Age at disease onset may help to further characterize the disease phenotype in psoriatic arthritis. Joint Bone Spine, v.83, n.5, p.533-537, 2016. DOI: https://doi.org/10.1016/j.jbspin.2015.09.004.

BARNAS, J. L.; RITCHLIN, C. T.. Etiology and Pathogenesis of Psoriatic Arthritis. Psoriatic Arthritis, v.41, n.4, p.643-663, 2015. DOI: https://doi.org/10.1016/i.rdc.2015.07.006.

CHOOI, Y.; C.; DING, C.; MAGKOS, F.. The epidemiology of obesity. Metabolism: Clinical and Experimental, v.92, p.610, 2019. DOI:

https://doi.org/10.1016/j.metabol.2018.09.005

COATES, L. C.; STRAND, V.; WILSON, H.; REVICKI, D.; STOLSHEK, B.; SAMAD, A.; CHUNG, J. B.; GLADMAN, D.; MEASE, P. J.. Measurement properties of the minimal disease activity criteria for psoriatic arthritis. RMD open, v.5, n.2, p.1-2, 2019. DOI: https://doi.org/10.1136/rmdopen2019-001002.

EDER, L.; A.; ROSEN, C. F.; CHANDRAN, V.; GLADMAN, D. D.. The Association Between Obesity and Clinical Features of Psoriatic Arthritis: a Case-control Study. The Journal of Rheumatology, v.44, n.4, p.437-443, 2017. DOI: https://doi.org/10.3899/jrheum.160532.

EDER, L.; HADDAD, A.; ROSEN, C. F.; LEE, K.; CHANDRAN, V.; COOK, R.; GLADMAN, D. D.. The Incidence and Risk Factors for Psoriatic Arthritis in Patients With Psoriasis: a Prospective Cohort Study. Arthritis \& Rheumatology, v.68, n.4, p.915-923, 2016. DOI:

https://doi.org/10.1002/art.39494.

EGEBERG, A.; SØRENSEN, J. A.; GISLASON, G. H.; KNOP, F. K.; SKOV, L.. Incidence and Prognosis of Psoriasis and Psoriatic Arthritis in Patients Undergoing Bariatric Surgery. JAMA surgery, v.152, n.4, p.344-349, 2017. DOI:

https://doi.org/10.1001/jamasurg.2016.4610.

FERGUSON, L. D.; BROWN, R.; CELIS-MORALES, C.; WELSH, P.; LYALL, D. M.; PELL, J. P.; MCINNES, L. B.; SIEBERT, S.; SATTAR, N.. Association of central adiposity with psoriasis, psoriatic arthritis and rheumatoid arthritis: a cross-sectional study of the UK Biobank. Rheumatology, v.58, n.12, p.21372142, 2019. DOI:

https://doi.org/10.1093/rheumatology/kez192.

HØJGAARD, P.; GLINTBORG, B.; KRISTENSEN, L. E.; GUDBJORNSSON, B.; LOVE, T. J.; DREYER, L.. The influence of obesity on response to tumour necrosis factor- $\alpha$ inhibitors in psoriatic arthritis: results from the DANBIO and ICEBIO registries. Rheumatology, v.55, n.12, p.2191-2199, 2016.
DOI: https://doi.org/10.1093/rheumatology/kew326.

KAINE, J.; SONG, X.; KIM, G.; HUR, P.; PALMER, J. B.. Higher Incidence Rates of Comorbidities in Patients with Psoriatic Arthritis Compared with the General Population Using U.S. Administrative Claims Data. Journal of Managed Care \& Specialty Pharmacy, v.25, n.1, p.122-132, 2019. DOI: https://doi.org/10.18553/jmcp.2018.17421.

KARREMAN, M. C.; WEEL, A. E. A. M.; VAN, D. V. M.; VIS, M.; TCHETVERIKOV, I.; NIJSTEN, T. E. C.; WAKKEE, M.; HAZES, J. M. W.; LUIME, J. J.. Prevalence of Psoriatic Arthritis in Primary Care Patients With Psoriasis. Arthritis \& Rheumatology, v.68, n.4, p.924-931, 2016. DOI: https://doi.org/10.1002/art.39530.

KAVANAUGH, A.; PAPP, K.; GOTTLIEB, A. B.; DE JONG, E. M. G. J.; CHAKRAVARTY, S. D.; KAFKA, S.; LANGHOLFF, W.; FARAHI, K.; SRIVASTAVA, B.; SCHER, J. U. D.. Baseline disease characteristics, and treatment history of psoriasis patients with self-reported psoriatic arthritis enrolled in the PSOLAR registry. BMC rheumatology, v.2, p.29, 2018. DOI: https://doi.org/10.1186/s41927-018-0034-7.

KLINGBERG, E.; BILBERG, A.; BJÖRKMAN, S.; HEDBERG, M.; JACOBSSON, L.; FORSBLAD-D'ELIA, H.; CARLSTEN, H.; ELIASSON, B.; LARSSON, I.. Weight loss improves disease activity in patients with psoriatic arthritis and obesity: an interventional study. Arthritis Research \& Therapy, v.21, n.1, p.11-17, 2019. DOI: https://doi.org/10.1186/s13075019-1810-5.

MAGLIO, C.; PELTONEN, M.; RUDIN, A.; CARLSSON, L. M. S.. Bariatric Surgery and the Incidence of Psoriasis and Psoriatic Arthritis in the Swedish Obese Subjects Study. PubMed, v.25, n.12, 2017. DOI: https://doi.org/10.1002/oby.21955.

QUEIRO, R.; LORENZO, A.; TEJÓN, P.; COTO, P.; PARDO, E. Obesity in psoriatic arthritis: comparative prevalence and associated factors. PubMed, v.98, n.28, jul 2019. DOI: https://doi.org/10.1097/MD.0000000000016400.

QUEIRO, R.; LORENZO, A.; TEJÓN, P.; PARDO, E.; COTO, P.; BALLINA, J.. Polyarticular evolution and late-onset psoriasis may be associated with cardiovascular disease in psoriatic arthritis. International Journal of Rheumatic Diseases, v.22, n.2, p.269-274, 2019. DOI: https://doi.org/10.1111/1756185X.13421.

RADNER, H.; LESPERANCE, T.; ACCORTT, N. A.; SOLOMON, D. H.. Incidence and Prevalence of Cardiovascular Risk Factors Among Patients With Rheumatoid Arthritis, Psoriasis, or Psoriatic Arthritis. Arthritis Care \& Research, v.69, n.10, p. 1510-1518, 2017. DOI: https://doi.org/10.1002/acr.23171. 
TALOTTA, R.; ATZENI, F.; SARZI-PUTTINI, P.; MASALA, I. F.. Psoriatic arthritis: From pathogenesis to pharmacologic management. Pharmacological Research, v.148, p.104-394, 2019. DOI: https://doi.org/10.1016/j.phrs.2019.104394.

WIBETOE, G.; IKDAHL, E. ; ROLLEFSTAD, S.; OLSEN, I. C.; BERGSMARK, K.; KVIEN, T. K.; SALBERG, A.; SOLDAL, D. M. ;
BAKLAND, G.; LEXBERG, ̊̊.; FEVANG, B. ; GULSETH, H. C . HAUGEBERG, G.; SEMB, A. G.. Cardiovascular disease risk profiles in inflammatory joint disease entities. Arthritis Research \& Therapy, v.19, n.1, p.153, 2017. DOI: https://doi.org/10.1186/s13075-017-1358-1.

A CBPC - Companhia Brasileira de Produção Científica (CNPJ: 11.221.422/0001-03) detém os direitos materiais desta publicação. Os direitos referem-se à publicação do trabalho em qualquer parte do mundo, incluindo os direitos às renovações, expansões e disseminações da contribuição, bem como outros direitos subsidiários. Todos os trabalhos publicados eletronicamente poderão posteriormente ser publicados em coletâneas impressas sob coordenação da Sustenere Publishing, da Companhia Brasileira de Produção Científica e seus parceiros autorizados. Os (as) autores (as) preservam os direitos autorais, mas não têm permissão para a publicação da contribuição em outro meio, impresso ou digital, em português ou em tradução. 